\title{
THE LANDSAT DATA CONTINUITY MISSION OPERATIONAL LAND IMAGER (OLI) SENSOR
}

\author{
Brian L. Markham ${ }^{1}$, Edward J. Knight ${ }^{2}$, Brent Canova ${ }^{2}$, Eric Donley ${ }^{2}$, Geir Kvaran ${ }^{2}$, Kenton Lee $^{2}$, \\ Julia A. Barsi ${ }^{3}$, Jeffrey A. Pedelty ${ }^{1}$, Philip W. Dabney ${ }^{1}$ and James R. Irons ${ }^{1}$ \\ ${ }^{1}$ NASA/GSFC, Greenbelt, MD 20771; ${ }^{2}$ Ball Aerospace \& Technology Corp., Boulder, CO 80301; \\ ${ }^{3}$ SSAI/GSFC, Greenbelt, MD 20771
}

\section{INTRODUCTION/STATUS}

The Landsat Data Continuity Mission (LDCM) is being developed by NASA and USGS and is currently planned for launch in January 2013 [1]. Once on-orbit and checked out, it will be operated by USGS and officially named Landsat-8. Two sensors will be on LDCM: the Operational Land Imager (OLI), which has been built and delivered by Ball Aerospace \& Technology Corp (BATC) and the Thermal Infrared Sensor (TIRS)[2], currently being built and tested at Goddard Space Flight Center (GSFC) with a planned delivery of Winter 2012. The OLI covers the Visible, Near-IR (NIR) and Short-Wave Infrared (SWIR) parts of the spectrum; TIRS covers the Thermal Infrared (TIR). This paper discusses only the OLI instrument and its pre-launch characterization; a companion paper covers TIRS.

\section{INSTRUMENT DESCRIPTION}

The OLI is an all-reflective pushbroom radiometer that images a $185 \mathrm{~km}$ swath like the whiskbroom TM and ETM+ instruments (Figure 1, 2). The OLI has 7 spectral bands (Table 1) that are similar to the reflective bands on the ETM+, though refined in bandwidth and location. In addition, it has a band centered at $443 \mathrm{~nm}$ that is intended to provide additional information about coastal regions and the atmosphere, and a band centered at 1373 $\mathrm{nm}$ for detection of cirrus clouds. As per the ETM+, all spectral bands, with the exception of the panchromatic band are 30m IFOV; the pan band is $15 \mathrm{~m}$. The dynamic range of OLI is set that it will not saturate for nonspecular targets across all solar zenith angles observed on orbit. OLI data are transmitted at 12-bit radiometric resolution.

The OLI is designed to achieve $5 \%$ uncertainty in radiance calibration and $3 \%$ uncertainty in reflectance calibration. To assist in maintaining this calibration, two full aperture Spectralon ${ }^{\mathrm{TM}}$ solar diffusers are part of the instrument as well as internal lamps. The solar diffusers will be used about every week, the lamps every day. The OLI will also observe the moon monthly. Detector to detector normalization within a band is required to be better than $0.5 \%$; the diffuser observations will be key in maintaining this relative calibration. 


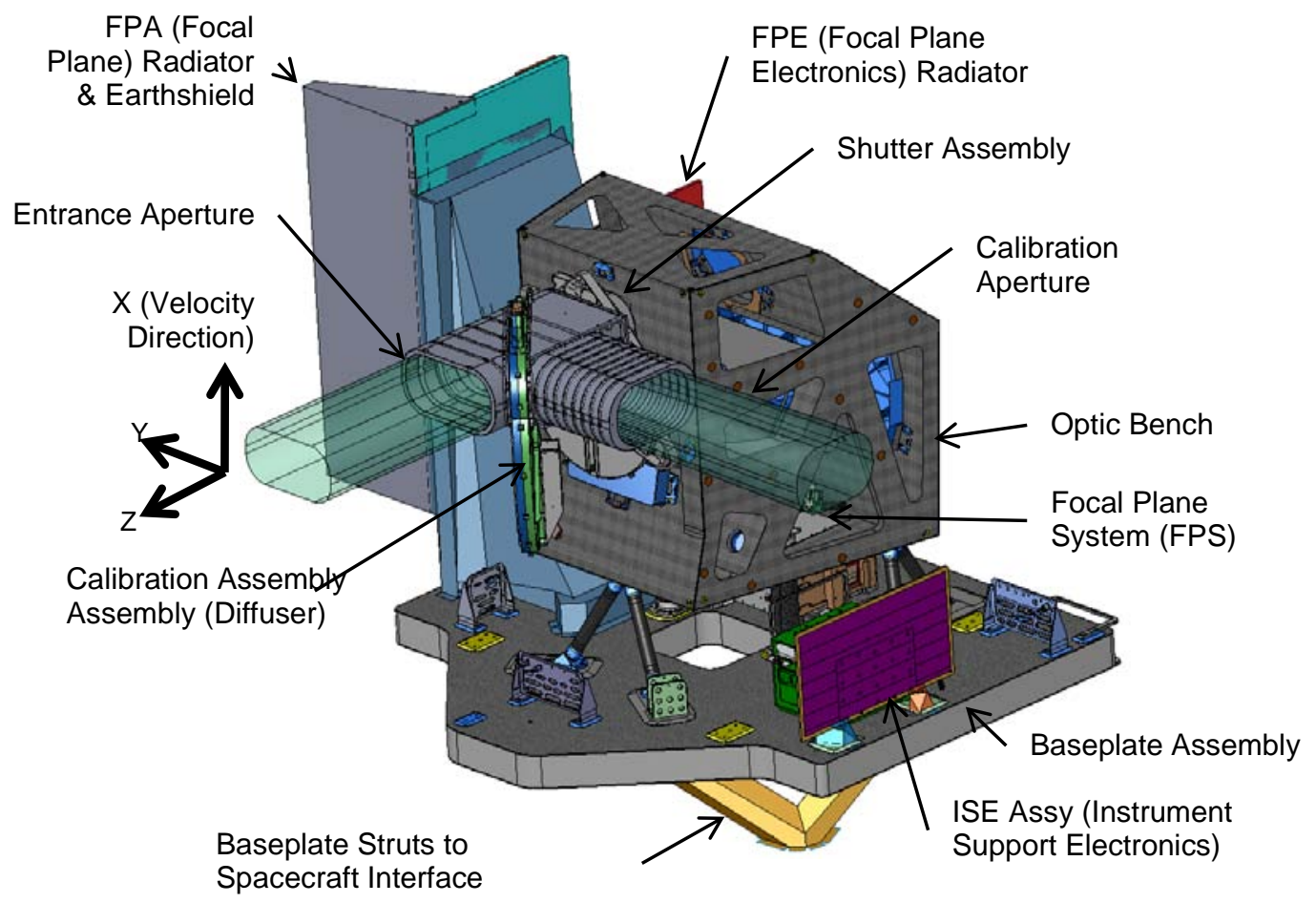

Figure 1. Operational Land Imager

3.

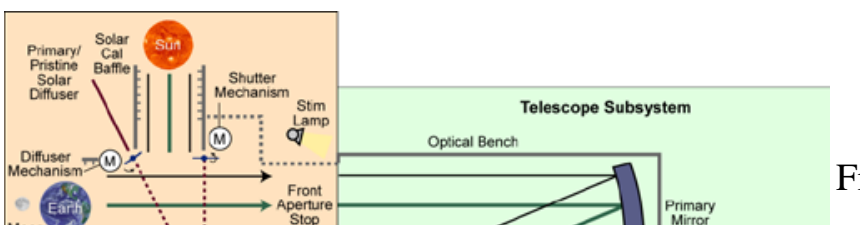

Table 1: OLI Spectral Bands

Figure 2. OLI Block Diagram

PRE-LAUNCH TESTING AND RESULTS

of

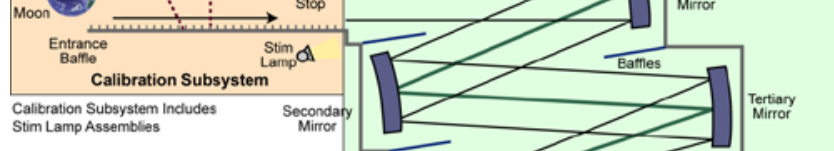

\subsection{Spatial/Geometry}

The principal spatial and geometric characterizations the OLI instrument were performed in a thermal vacuum chamber using the BATC horizontal collimator assembly (HCA). The HCA includes various targets at the focus of the collimator that are used to test edge response, bright target recovery, ghosting and line of sight. Edge response as opposed

to

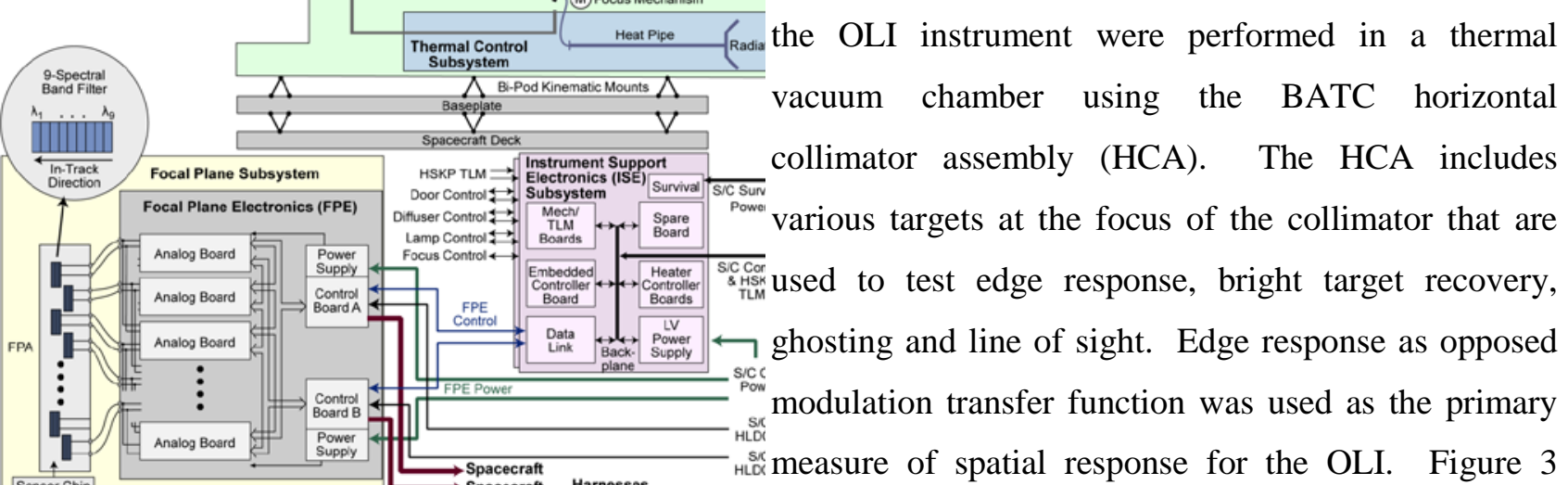

shows samples of the OLI edge response, where all requirements were met. 


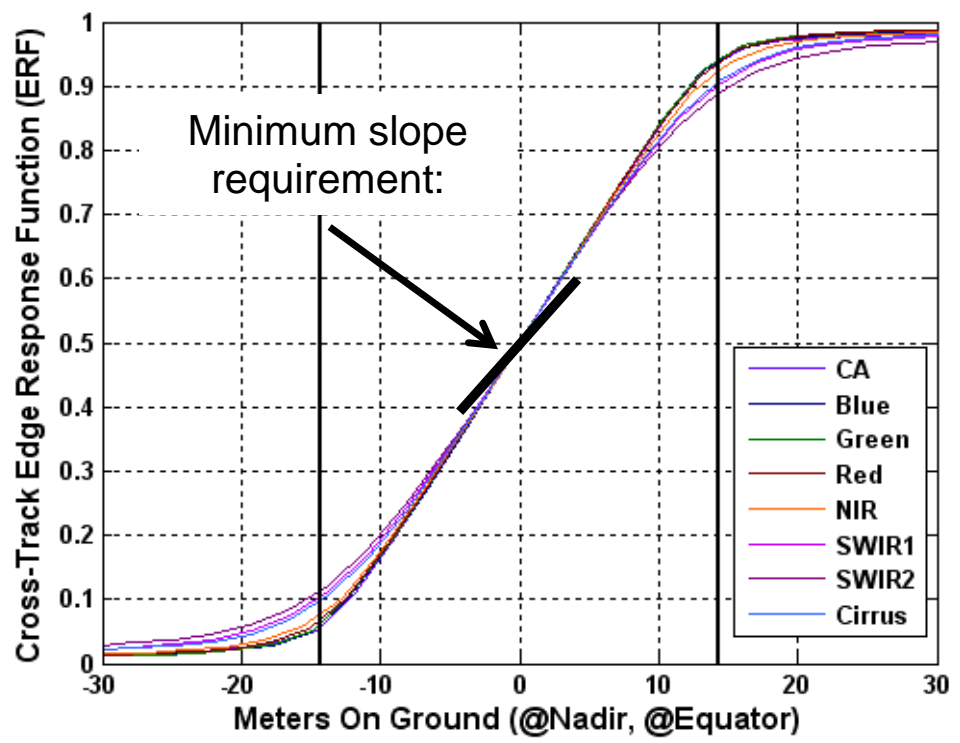

Figure 3. OLI edge response for the 30-meter band

\subsection{Spectral/Radiometry}

Spectral and radiometric testing of the OLI was performed at BATC with the OLI in a thermal vacuum chamber and with the spectral and radiometric sources outside. Testing included in-band relative spectral response, linearity, stability, noise, and absolute calibration. Band average relative spectral responses are shown in Figure 4, and have been released to the web: http://ldcm.nasa.gov/spacecraft instruments/oli band average.html. In Figure 5 are shown the median Signal to Noise ratios of the OLI bands at typical radiance levels $\left(\mathrm{L}_{\text {typ }}\right)$, i.e., about a $10 \%$ reflectance at a $45^{\circ}$ solar zenith angle.

\section{SUMMARY}

The OLI for LDCM has been completed, delivered and integrated onto the LDCM spacecraft. It meets the vast majority of its performance requirements with a few minor exceptions. 


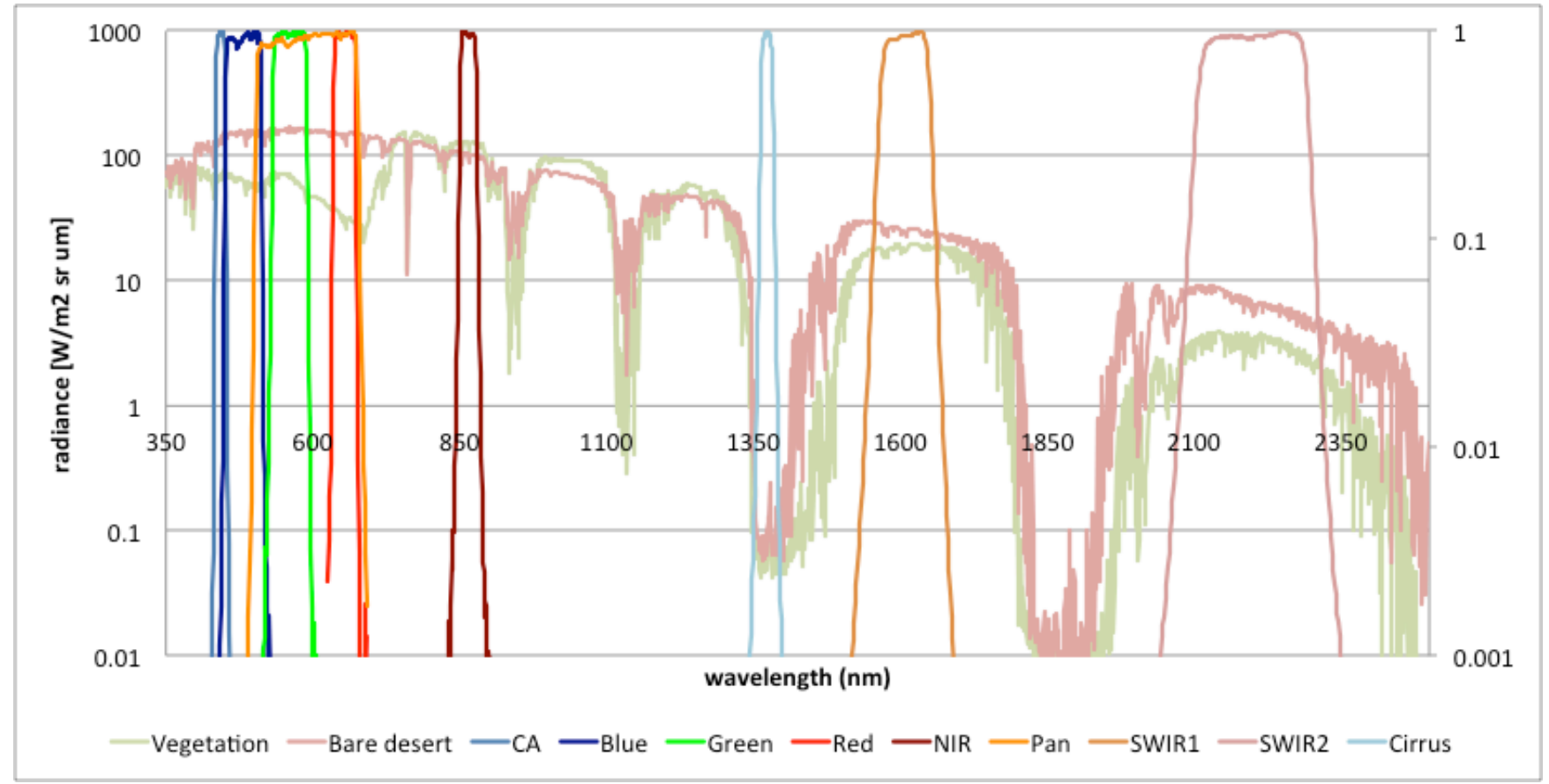

Figure 4. OLI band average relative spectral responses.

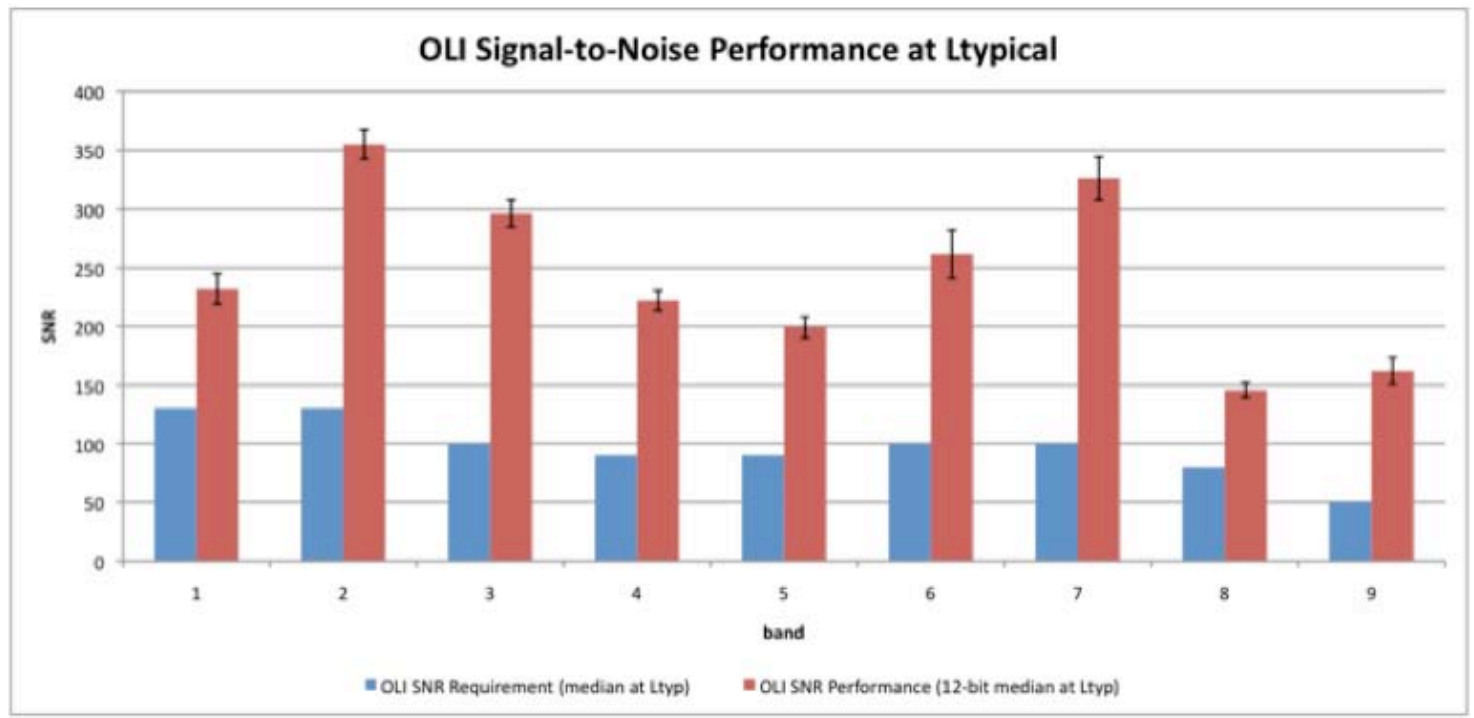

Figure 5. OLI Signal to Noise Performance

\section{REFERENCES}

[1] J.R. Irons and J.L. Dwyer, “An Overview of the Landsat Data Continuity Mission,” Proceedings of the SPIE, Vol. 7695,12 May 2010. [2] D. Reuter et al., “The Thermal Infrared Sensor on the Landsat Data Continuity Mission,” IGARSS 2011, p 754-757, Honolulu, HI. 